\title{
Lozenge Dosage Form
}

National Cancer Institute

\section{Source}

National Cancer Institute. Lozenge Dosage Form. NCI Thesaurus. Code C42955.

A solid composed of active and/or inert ingredient(s), sweeteners, other flavorings and mucilage, intended for oral administration. 